\title{
Differential risk of death in older residents in nursing homes prescribed specific antipsychotic drugs: population based cohort study
}

\author{
(C) $\mathbb{( 1 )}$ OPEN ACCESS
}

\author{
K F Huybrechts instructor in medicine ${ }^{1}, \mathrm{~T}$ Gerhard assistant professor ${ }^{2}$, S Crystal board of governors \\ professor $^{2}, \mathrm{M}$ Olfson professor of clinical psychiatry ${ }^{3}$, J Avorn professor of medicine ${ }^{1}$, R Levin \\ programmer $^{1}$, J A Lucas assistant research professor ${ }^{4}$, S Schneeweiss associate professor of \\ medicine $^{1}$
}

'Division of Pharmacoepidemiology and Pharmacoeconomics, Department of Medicine, Brigham and Women's Hospital and Harvard Medical School, Boston 02120, MA, United States; ${ }^{2}$ Ernest Mario School of Pharmacy, Rutgers University, New Brunswick, NJ 08901; ${ }^{3}$ Columbia University, New York, NY 10032; ${ }^{4}$ Institute for Health, Health Care Policy and Aging Research, Rutgers University, New Brunswick, NJ 08901

\begin{abstract}
Objective To assess risks of mortality associated with use of individual antipsychotic drugs in elderly residents in nursing homes.

Design Population based cohort study with linked data from Medicaid, Medicare, the Minimum Data Set, the National Death Index, and a national assessment of nursing home quality.

Setting Nursing homes in the United States.

Participants 75445 new users of antipsychotic drugs (haloperidol, aripiprazole, olanzapine, quetiapine, risperidone, ziprasidone). All participants were aged $\geq 65$, were eligible for Medicaid, and lived in a nursing home in 2001-5.
\end{abstract}

Main outcome measures Cox proportional hazards models were used to compare 180 day risks of all cause and cause specific mortality by individual drug, with propensity score adjustment to control for potential confounders.

Results Compared with risperidone, users of haloperidol had an increased risk of mortality (hazard ratio $2.07,95 \%$ confidence interval 1.89 to 2.26$)$ and users of quetiapine a decreased risk $(0.81,0.75$ to $0.88)$. The effects were strongest shortly after the start of treatment, remained after adjustment for dose, and were seen for all causes of death examined. No clinically meaningful differences were observed for the other drugs. There was no evidence that the effect measure modification in those with dementia or behavioural disturbances. There was a dose-response relation for all drugs except quetiapine.

Conclusions Though these findings cannot prove causality, and we cannot rule out the possibility of residual confounding, they provide more evidence of the risk of using these drugs in older patients, reinforcing the concept that they should not be used in the absence of clear need. The data suggest that the risk of mortality with these drugs is generally increased with higher doses and seems to be highest for haloperidol and least for quetiapine.

\section{Introduction}

Up to a third of all elderly patients in nursing homes are treated with antipsychotic drugs. ${ }^{1-5}$ In the past, inappropriate prescribing of antipsychotics in nursing homes has primarily been considered a marker of suboptimal care. ${ }^{67}$ Federal action thus focused primarily on defining and enforcing specific diagnostic criteria for the initiation and monitoring of these drugs (Omnibus Budget Reconciliation Act). ${ }^{8}$ In recent years, evidence has accumulated that their use is a drug safety issue as well. After earlier warnings of increased risks of cerebrovascular events (with risperidone, olanzapine, and aripiprazole), ${ }^{9}$ the Food and Drug Administration issued an advisory warning in 2005 that atypical antipsychotics were associated with a $60-70 \%$ increased risk of death compared with placebo in randomised controlled trials among older patients with dementia, and black box warnings were added to the labels of all atypical drugs. ${ }^{10}$ Subsequent studies found risks at least as high among users of conventional antipsychotics, ${ }^{11-13}$ and the Food and Drug Administration issued a similar warning for such drugs in 2008. ${ }^{14}$ Despite these strong safety warnings, use of antipsychotic drugs in nursing homes is likely to remain substantial-as evidenced by the recent audit by the US Department of Health and Human Services ${ }^{15}$ - because of the continued growth in the number of 
people with dementia, the perceived need for some type of intervention in patients with severe persistent symptoms, and a paucity of effective alternative pharmacological or behavioural approaches. ${ }^{16}$ Questions about the comparative safety of individual antipsychotic drugs are therefore of paramount importance to patients and prescribers, but the existing Food and Drug Administration advisories do not distinguish between drugs in these classes and thus offer no guidance in that regard.

In the absence of randomised trials, surveillance data from large longitudinal healthcare databases provide a unique opportunity to examine the comparative safety of individual drugs, provided that rigorous methodological approaches are applied to minimise bias. Such databases also include the most vulnerable segments of the population, such as residents of nursing homes, who are commonly excluded from trials.

We investigated whether the risk of overall and cause specific mortality is equal across antipsychotic drugs or whether there are regimens with safety advantages that should be prescribed preferentially in older residents of nursing homes.

\section{Methods}

\section{Data source and study cohort}

The study cohort was drawn from a merged dataset of Medicaid and Medicare claims, the minimum data set (MDS), the Online Survey Certification and Reporting (OSCAR) system, and the National Death Index in 45 states in the United States (all except Arizona, Delaware, Nevada, Oregon, and Rhode Island) for 2001-5. Claims data provided information on patients' demographics, eligibility for Medicaid, physician services and admissions to hospital and their accompanying diagnoses, admissions to long term care, and filled prescriptions for drugs. The Minimum Data Set is a federally mandated health assessment tool used in nursing homes that captures information on physical, psychological, and psychosocial functioning, active clinical diagnoses, health conditions, treatments, and services. The Online Survey Certification and Reporting system is a uniform database of nursing home regulatory reviews, which is generated yearly for all nursing homes certified by the Centers for Medicare and Medicaid Services (CMS) and includes operational and staffing characteristics and aggregate resident characteristics.

Our cohort consisted of all patients aged $\geq 65$ who were dually eligible for Medicare and Medicaid, who started treatment with an antipsychotic drug during a stay in a nursing home, and who had six months' continuous Medicaid coverage before the date they started the antipsychotic drug (index date). Incident use required the absence of filled prescriptions for an antipsychotic in the six months before the index date. We used an incident user design to avoid underascertainment of events that occur soon after treatment begins ${ }^{17}$ and to ensure that baseline covariates at study entry were assessed before the start of treatment and were not affected by the treatment itself. ${ }^{18}$ Residents were excluded if they filled a prescription for both conventional and atypical antipsychotics on the index date and if they had a pre-existing diagnosis for cancer, schizophrenia, or bipolar disorder as these residents probably received antipsychotics for reasons other than behavioural problems related to dementia (fig $1 \Downarrow$ ).

\section{Exposure to antipsychotic drugs}

Antipsychotic drugs considered include haloperidol, aripiprazole, olanzapine, quetiapine, risperidone, and ziprasidone. Other drugs (thioridazine, chlorpromazine, perphenazine, fluphenazine, acetophenazine, mesoridazine, promazine, trifluoperazine, triflupromazine, chlorprothixene, loxapine, molindone, pimozide, and thiothixene) were excluded because they were used by too few patients ( $1 \%$ for all combined) to permit interpretable estimation of the associations between drug and outcome (fig $1 \Downarrow$ ). Each participant was assigned to a specific drug based on the first prescription; a given exposure was considered as discontinued if there was a gap in treatment of 14 days or more. We chose the most commonly used drug in this population, risperidone, as the reference drug. We converted daily doses of the index drug to equivalent doses of chlorpromazine (in $\mathrm{mg}$ ) ${ }^{19-21}$ and used the median daily dose in the population $(50 \mathrm{mg})$ as a cut off to assess the effect in groups of higher and lower doses (see table A in appendix on bmj.com). In dose-response analyses, we empirically defined cut offs for low, medium, and high dose groups for each individual drug after inspection of the dose distributions (see table B in appendix on bmj.com).

\section{Outcomes}

Information on vital status and causes of death was available through linkage with the National Death Index. We identified all deaths in the cohort within 180 days after the index date. We excluded deaths from cancer because such cancers were probably pre-existing and might have been associated with use of some drugs in this class to reduce nausea or to potentiate drugs for pain relief. As we recognise the potential for misclassification of causes of death in older populations, ${ }^{22}$ we examined the following groups of causes based on suggested pharmacological effects of antipsychotics ${ }^{23-27}$ and the Food and Drug Administration analysis ${ }^{10}$ : diseases of the circulatory system (ICD-10 (international classification of diseases, 10th revision) codes I00-I99), cerebrovascular diseases (ICD-10 codes I60-I69), diseases of the respiratory system (ICD10 codes J00-J99), and all other causes combined.

\section{Characteristics of patients and nursing homes}

We assessed characteristics of patients in the six months before cohort entry. Sociodemographic characteristics included age, sex, race, education, and geographical region (state). Clinical characteristics were determined according to the most recent Minimum Data Set assessment before the start of treatment, ICD-9 diagnostic and procedure codes associated with admissions to hospital and visits to physicians, and drug use. These variables assessed psychiatric morbidity, cardiovascular morbidity, cerebrovascular disease, Parkinson's disease, epilepsy, diabetes, obesity, functional impairment, the Charlson index, and use of healthcare services potentially predictive of adverse health outcomes in the short term (number of days in hospital, number of distinct prescriptions for drugs excluding antipsychotics).$^{28}$ We obtained characteristics of nursing homes, which could be correlated with care processes and risk of adverse health outcomes, from the Online Survey Certification and Reporting system. These included variables such as facility size, occupancy rate, availability of special care units, staffing levels, ownership, resident characteristics (for example, proportion with dementia, depression) and quality indicators (for example, proportion bedbound).

\section{Data analysis}

We compared distributions of sociodemographic, clinical, and use characteristics among participants who started taking different antipsychotics and calculated rates of mortality during follow-up. We censored follow-up at the time of discontinuation 
of treatment, augmentation, or switch to a different drug, and admission to hospital for 10 days or more, as treatment status is unknown during inpatient stays. To reduce the potential for informative censoring, we implemented a 30 day grace period. All deaths occurring during this time were attributed to the initial exposure.

We fitted proportional hazards models for pairwise comparisons against risperidone (unadjusted; adjusted for age, sex and calendar year; and adjusted for multiple variables). In multivariate analyses, we used propensity score adjustment to balance potential confounders. ${ }^{29}$ Propensity scores were derived from predicted probabilities of the start of treatment estimated in logistic regression models that contained all covariates listed above. Cox models were stratified across 10ths of the propensity score. In addition, we plotted multivariate adjusted

Kaplan-Meier curves for survival as a function of the duration of use of the index antipsychotic using inverse probability of treatment weighting. ${ }^{30}$ In confirmatory analyses, we fitted high dimensional propensity scores, ${ }^{31}$ which have been shown to improve validity in claims data studies by further reducing residual confounding. ${ }^{32} 33$

We ran propensity score and outcome models separately in groups defined by recorded diagnoses of dementia and behavioural disturbances and by dose, dividing each group into those taking $\leq 50 \mathrm{mg}$ or $>50 \mathrm{mg}$ chlorpromazine equivalents a day. A dose-response analysis was conducted to compare the risk of deaths from causes other than cancer in residents treated with high or medium versus low dose antipsychotics, for all drugs separately and combined. In sensitivity analyses, we estimated the strength of the residual confounding that would be required to fully explain the observed associations for deaths from causes other than cancer if in truth no such associations existed. $^{34}$

\section{Results}

From 2001 to 2005, 75445 older residents of nursing homes started treatment with antipsychotic drugs. Compared with the reference group risperidone, patients who started taking haloperidol were more likely at baseline to have cardiovascular disease, less likely to have psychiatric comorbidities, and had slightly worse general indicators of comorbidity. Patients who started taking risperidone had slightly fewer recorded diagnoses of depression and less use of antidepressants and other psychoactive drugs than patients who started treatment with other atypical antipsychotics. They also tended to have a lower use of cholinesterase inhibitors. Patients treated with quetiapine had more diagnoses of parkinsonism than the other groups, and patients treated with olanzapine had fewer diagnoses of diabetes. There were also some differences in the prescribing of specific drugs between regions and settings (table $1 \Downarrow$ and table $C$ in appendix on bmj.com).

Eighty per cent of residents ( $\mathrm{n}=60167)$ were censored before the end of 180 days. Discontinuation of treatment was the most common reason for censoring $(57.2 \%, n=34388)$, followed by hospital admission for 10 days or longer $(17.4 \%, \mathrm{n}=10464)$, treatment switches or augmentation $(13.1 \%, \mathrm{n}=7902)$, and death $(12.3 \%, \mathrm{n}=7413)$. A total of 6598 residents died from causes other than cancer during the first 180 days after the start of treatment, yielding an event rate of 37.1 per 100 person years (95\% confidence interval 36.2 to 38.0 ). Table 2 shows event rates for specific treatments $\Downarrow$.

\section{All cause mortality}

Findings from the Cox regression analyses indicated that, compared with risperidone, patients treated with haloperidol had double the risk of mortality (hazard ratio adjusted for propensity score $2.07,95 \%$ confidence interval 1.89 to 2.26 ) and patients treated with quetiapine had a reduced risk $(0.81$, 0.75 to 0.88 ). No meaningful differences in risk were observed for aripiprazole, olanzapine, and ziprasidone (table $3 \Downarrow$ ). Adjusted Kaplan-Meier plots are consistent with these findings (fig $2 \Downarrow$ ). The effect of haloperidol was strongest during the first 40 days of treatment (hazard ratio adjusted for propensity score 2.34, 2.11 to 2.60$)$ and reduced to 1.32 (1.02 to 1.71$)$ and $1.46(1.07$ to 2.00) after 40-79 and 80-180 days of treatment, respectively. The corresponding rate ratios for quetiapine were 0.74 (0.66 to 0.82 ), 0.87 (0.75 to 1.01 ), and 0.91 (0.79 to 1.05$)$. Analyses stratified by dose confirmed the overall findings (figs $3 \Downarrow$ and $4 \Downarrow$ ), and we did not find evidence that the effect measure was modified by the presence of a recorded diagnosis of dementia or behavioural disturbances (see table D in appendix on bmj.com). Sensitivity analyses indicated that for an unmeasured confounder (such as frailty, severity of dementia) present in $25 \%$ of the population, relative risks $\geq 5$.0 linking the hypothetical confounder to both haloperidol use and mortality would be needed to fully explain the observed association with mortality. For confounders present in $10 \%$ or $5 \%$ of the population, relative risks of $>6.0$ and 7.5 , respectively, would be needed. To fully explain the protective association for quetiapine, relative risks of $>2.5,3.5$, and 5.0 would be required for an unmeasured confounder present in $25 \%, 10 \%$, and $5 \%$, respectively, of the population (see figs $\mathrm{A}$ and $\mathrm{B}$ in appendix on bmj.com).

\section{Cause specific mortality}

Almost half $(49 \%, n=3262)$ of deaths were recorded as caused by circulatory disorders, $10 \%(\mathrm{n}=655)$ by cerebrovascular diseases, and $15 \%(n=981)$ by respiratory disorders. Table 2 shows the cause specific event rates by treatment group $\Downarrow$. The increased risk of death with haloperidol and the decreased risk with quetiapine were observed for all causes examined. Although no difference in overall mortality was observed for olanzapine, findings suggest a possibly lower risk of death from cerebrovascular diseases (hazard ratio adjusted for propensity score $0.88,0.73$ to 1.07 ; hazard ratio adjusted for high dimensional propensity score $0.83,0.68$ to 1.01 ). Given the relatively small number of patients treated with aripiprazole and ziprasidone, the associations for cause specific mortality are imprecisely estimated (table $3 \Downarrow$ ).

When we compared the risk of all deaths from causes other than cancer in residents treated with high versus low and medium versus low dose of a given drug, our findings suggest a dose-response relation for all antipsychotic drugs except quetiapine (fig $4 \Downarrow$ ). The dose effects were most pronounced for haloperidol (hazard ratio adjusted for propensity score 1.84 for high and 1.40 for medium dose, both compared with low dose) and for risperidone (1.35 for high and 1.19 for medium dose).

\section{Discussion}

\section{Summary of main findings}

In elderly residents receiving antipsychotic drugs in nursing homes, there is variation in the risk of death according to the type of drug used. Compared with risperidone users, haloperidol users had an increased risk and quetiapine users had a decreased risk of dying within 180 days. The effects were strongest shortly 
after the start of treatment and remained after adjustment for dose. No clinically meaningful differences were observed for the other drugs. There was no evidence that the treatment effect differed for patients with a diagnosis of dementia or behavioural disturbances. A dose-response relation was observed for all drugs, except quetiapine. The study was carried out in 75445 residents of nursing homes in 45 states in the US. All participants were aged $\geq 65$, started treatment with antipsychotic drugs between 2001 and 2005, and were eligible for Medicaid.

\section{Results in context}

Our results for haloperidol are consistent with the findings of observational studies that report a greater risk of death with conventional versus atypical antipsychotics in older adults, ${ }^{11-13} 32$ and the study by Liperoti et al, which found an increased risk with haloperidol compared with risperidone in patients with dementia in nursing homes in five US states $(n=9729) .{ }^{35}$ Our finding of a reduced risk of death from causes other than cancer with quetiapine is compatible with an observation made by Rossom et al in a population of predominately male veterans with dementia. ${ }^{36}$ In contrast, Liperoti et al, using Minimum Data Set information to determine drug exposure, did not observe differences in mortality between individual atypical drugs. ${ }^{35}$

Another study that examined causes of death in elderly users of antipsychotics was done in British Columbia and found that those who started treatment with conventional rather than atypical antipsychotics had the highest relative risk of death from respiratory diseases (hazard ratio $1.71,1.35$ to 2.17 ), followed by circulatory disorders $(1.23,1.10$ to 1.36$) .{ }^{37}$ Our findings for haloperidol compared with risperidone in nursing home residents in the US mirror these results. Differences in cause specific mortality with atypical antipsychotic drugs have not previously been examined. Our comparative findings, however, are consistent with results reported in a recent meta-analysis that combined data from placebo controlled trials and found that the risk of cardiovascular outcomes in elderly patients with dementia was increased with risperidone and olanzapine but not with quetiapine. Likewise, risperidone, but not olanzapine or quetiapine, was found to be associated with an increased risk of stroke. ${ }^{38}$

\section{Study strengths and weaknesses}

A major strength of our study is the large population of residents in US nursing homes, which allowed us to examine the effect of individual drugs, different doses, and cause specific mortality. It was large enough to allow us to restrict our population to new users, which reduces the likelihood of missing early adverse events, allows for an evaluation of risks over duration of use, ensures that the assessment of baseline characteristics is not influenced by any effects of antipsychotic treatment, and reduces the likelihood that current treatment assignment is influenced by past experiences related to drugs, such as adverse events and refractory symptoms. ${ }^{17}$ To clearly identify exposure groups, we compared single drug treatments with each other and censored patient follow-up as soon as the patient switched drugs or augmented treatment with a second antipsychotic drug.

We looked at residual confounding by indication as an alternative explanation of our findings. This would occur if patients who were frail and at increased risk of death were more or less likely to be prescribed certain antipsychotic drugs. We supplemented confounder information derived from claims data with clinical assessment data (Minimum Data Set) and potential indicators of nursing home quality (Online Survey Certification and Reporting). ${ }^{39}$ We used multiple methods to mitigate confounding by the predefined covariates and by proxies for unobserved factors (adjustment for high dimensional propensity score $^{31}$ ) and found results to be consistent. Sensitivity analyses showed that strong risk factors for death that are fairly imbalanced among exposure groups must be unmeasured and uncontrolled to explain the observed associations for deaths from causes other that cancer.

There is potential for misclassification of exposure status through lack of consumption of filled prescriptions. Patients in nursing homes are closely monitored, however, and adherence will be high, but occasional as-needed use might be an important source of misclassification. Patients were classified into groups with low and high doses according to the initial prescription. In case of dose adjustments, this could have resulted in misclassification of exposure in dose stratified analyses. Dose assignment, however, remained unchanged for over $90 \%$ of patients according to the second and last prescription filled before the end of follow-up. Results were also consistent when we used an alternative dose conversion algorithm. ${ }^{40}$

Broad categories of causes of death identified from death certificates have been found to agree with causes of death adjudicated by a physician, but problems arise when more specific subcategories of death-such as specific cardiovascular causes-are used.22 ${ }^{23}$ Although we examined only broad categories of causes of death, potential for misclassification remains, particularly in elderly people for whom the incidence of cardiovascular deaths tends to be overestimated, ${ }^{22}$ and we caution against overinterpretation of these aspects of the results. Our study population consisted of patients eligible for Medicaid. This restriction should not affect the validity of our findings. The central issue determining internal validity is comparability between the subcohorts included, not whether the study sample is representative of patients in nursing homes overall. As long as socioeconomic status and its correlates do not modify the effect of antipsychotic drugs on short term mortality, the findings should also be generalisable (that is, externally valid).

\section{Conclusions and implications}

Various environmental, psychosocial, and behavioural interventions have been developed to deal with behavioural disturbances in patients with dementia, ${ }^{41}$ but their effectiveness has not been rigorously studied ${ }^{42}$ and their implementation is often hampered by a lack of resources. ${ }^{43}$ Although such non-pharmacological interventions are nearly universally recommended as first line treatment,${ }^{44}$ they are commonly insufficient in people with severe and persistent or recurrent symptoms. Consequently, most patients will be given a psychotropic drug at some point in their disease progression. ${ }^{9}{ }^{16}$ In the absence of proved effective and safe alternative pharmacological treatments, it is likely that antipsychotic drugs will continue to be used widely, despite the fact that they have not been approved for this indication, their use cannot be justified as evidence based, ${ }^{38}$ and there are clear data confirming their associated risk.

The evidence provided in our study reinforces the important risks associated with the use of these drugs and underscores the need to try alternative means of dealing with behavioural problems in older patients with dementia. While our findings cannot tackle the efficacy-safety trade off involved in the decision to proceed with drug treatment for severe and refractory behavioural problems in people with dementia, they can contribute to decision making regarding treatment for clinicians considering use of antipsychotics in patients whose behavioural problems might themselves pose a risk to the patient or others. 
If the clinician faces a situation in which use of these drugs seems inevitable, our findings underscore the importance of always prescribing the lowest possible dose and of closely monitoring patients, especially shortly after the start of treatment. The evidence accumulated so far implies that use of haloperidol in this vulnerable population cannot be justified because of the excess harm. Quetiapine might be somewhat safer than other atypical drugs, but these findings will require replication in other studies.

Contributors: All authors were responsible for study concept and design. SC and SS acquired the data, which were analysed and interpreted by all authors. KFH drafted the manuscript, which was critically revised for important intellectual content by all authors. KFH, TG, RL, and SS did the statistical analysis. SC, JA, and SS gave administrative, technical, or material support. SS was the study supervisor, and KFH is guarantor. Funding: This study was supported by AHRQ/FDA Award HS017918 and AHRQ Award HS016097. KFH and SS were partially funded by National Institute of Mental Health R01-MH078708. The funders had no role in the design and conduct of the study; collection, management, analysis, and interpretation of the data; and preparation, review, or approval of the manuscript.

Competing interests: All authors have completed the ICMJE uniform disclosure form at www.icmje.org/coi_disclosure.pdf (available on request from the corresponding author) and declare: no support from any organisation for the submitted work; no financial relationships with any organisations that might have an interest in the submitted work in the previous three years; no other relationships or activities that could appear to have influenced the submitted work.

Ethical approval: This study was approved by the Institutional Review Board of the Brigham and Women's Hospital, the Partners Human Research Committee.

Data sharing: No additional data available.

1 Liperoti R, Mor V, Lapane KL, Pedone C, Gambassi G, Bernabei R. The use of atypical antipsychotics in nursing homes. J Clin Psychiatry 2003;64:1106-12.

2 Briesacher BA, Limcangco MR, Simoni-Wastila L, Doshi JA, Levens SR, Shea DG, et al. The quality of antipsychotic drug prescribing in nursing homes. Arch Intern Med 2005;165:1280-5.

3 Bronskill SE, Anderson GM, Sykora K, Wodchis WP, Gill S, Shulman KI, et al. Neuroleptic drug therapy in older adults newly admitted to nursing homes: incidence, dose, and specialist contact. J Am Geriatr Soc 2004;52:749-55.

4 Rochon PA, Stukel TA, Bronskill SE, Gomes T, Sykora K, Wodchis WP, et al. Variation in nursing home antipsychotic prescribing rates. Arch Intern Med 2007;167:676-83.

5 Chen Y, Briesacher B, Field T, Tjia J, Lau D, Gurwitz J. Unexplained variation across US nursing homes in antipsychotic prescribing rates. Arch Intern Med 2010;170:89-95.

6 Beers M, Avorn J, Soumerai S, Everitt D, Sherman D, Salem S. Psychoactive medication use in intermediate-care facility residents. JAMA 1988;260:3016-20.

7 Ray WA, Federspiel CF, Schaffner W. A study of antipsychotic drug use in nursing homes: epidemiologic evidence suggesting misuse. Am J Public Health 1980;70:485-91.

8 Avorn J, Wang P. Drug prescribing, adverse reactions, and compliance in elderly patients. In: Salzman C, ed. Clinical geriatric psychopharmacology. 4th ed. Lippincot, Williams \& Wilkins, 2005:23-47.

9 Jeste DV, Blazer D, Casey D, Meeks T, Salzman C, Schneider L, et al. ACNP White Paper: update on use of antipsychotic drugs in elderly persons with dementia. Neuropsychopharmacology 2007;33:957-70.

10 FDA Public Health Advisory. Deaths with antipsychotics in elderly patients with behavioral disturbances. 2010. www.fda.gov/drugs/drugsafety/

postmarketdrugsafetyinformationforpatientsandproviders/ucm053171

11 Wang PS, Schneeweiss S, Avorn J, Fischer MA, Mogun H, Solomon DH, et al. Risk of death in elderly users of conventional vs atypical antipsychotic medications. N Engl J Med 2005;353:2335-41.

12 Schneeweiss S, Setoguchi S, Brookhart A, Dormuth C, Wang PS. Risk of death associated with the use of conventional versus atypical antipsychotic drugs among elderly patients. CMAJ 2007;176:627-32.

13 Gill SS, Bronskill SE, Normand S-LT, Anderson GM, Sykora K, Lam K, et al. Antipsychotic drug use and mortality in older adults with dementia. Ann Intern Med 2007;146:775-86.

14 FDA. Information for Healthcare Professionals-antipsychotics. 2011. www.fda.gov/Drugs/ DrugSafety/PostmarketDrugSafetylnformationforPatientsandProviders/ucm124830 htm.

15 Office of Inspector General, Department of Health and Human Services. Medicare atypica antipsychotic drug claims for elderly nursing home residents. Department of Health and Human Services, 2011.
16 Wang P, Brookhart M, Setoguchi S, Patrick A, Schneeweiss S. Psychotropic medication use for behavioral symptoms of dementia. Curr Neurol Neurosci Rep 2006:6:490-5.

17 Ray WA. Evaluating medication effects outside of clinical trials: new-user designs. Am J Epidemiol 2003;158:915-20.

18 Schneeweiss S. A basic study design for expedited safety signal evaluation based on electronic healthcare data. Pharmacoepidemiol Drug Saf 2010;19:858-68.

19 Atkins M, Burgess A, Bottomley C, Riccio M. Chlorpromazine equivalents: a consensus of opinion for both clinical and research applications. Psychiatr Bull R Coll Psychiatr 1997;21:224-6.

20 Lehman A, Steinwachs D. Translating research into practice: the Schizophrenia Patient Outcomes Research Team (PORT) treatment recommendations. Schizophr Bull 1998;24:1-10.

21 Woods S. Chlorpromazine equivalent doses for the newer atypical antipsychotics. J Clin Psychiatry 2003;64:663-7.

22 Sesso H, Gaziano J, Glynn R, Buring J. Value of an endpoints committee versus the use of nosologists for validating cause of death. Contemp Clin Trials 2006;27:333-9.

23 Chan Y, Pariser S, Neufeld G. Atypical antipsychotics in older adults. Pharmacotherapy 1999;19:811-22.

24 Lawlor B. Behavioral and psychological symptoms in dementia: the role of atypical antipsychotics. J Clin Psych 2004:65:5-10.

25 Maixner S, Mellow A, Tandon R. The efficacy, safety, and tolerability of antipsychotics in the elderly. J Clin Psych 1999;60:29-41.

26 Tariot $\mathrm{P}$. The older patient: the ongoing challenge of efficacy and tolerability. J Clin Psych 1999;60:29-33.

27 Alexopoulos G, Streim J, Carpenter D, Docherty J. Expert Consensus Panel for Using Antipsychotic Drugs in Older Patients. Using antipsychotic agents in older patients. J Clin Psych 2004:65:5-99.

28 Schneeweiss S, Seeger JD, Maclure M, Wang PS, Avorn J, Glynn RJ. Performance of comorbidity scores to control for confounding in epidemiologic studies using claims data. Am J Epidemiol 2001;154:854-64.

29 Braitman LE, Rosenbaum PR. Rare outcomes, common treatments: analytic strategies using propensity scores. Ann Intern Med 2002;137:693-5.

30 Robins J, Hernán M, Brumback B. Marginal structural models and causal inference in epidemiology. Epidemiology 2000;11:550-60.

31 Schneeweiss S, Rassen J, Glynn R, Avorn J, Mogun H, Brookhart M. High-dimensional propensity score adjustment in studies of treatment effects using health care claims data. Epidemiology 2009;20:512-22.

32 Huybrechts K, Rothman K, Silliman R, Brookhart M, Schneeweiss S. Risk of death and hospital admission for major medical events after initiation of psychotropic medications in older adults admitted to nursing homes. CMAJ 2011;183:E411-9.

33 Patorno E, Bohn R, Wahl P, Avorn J, Patrick A, Liu J, et al. Anticonvulsant medications and the risk of suicide, attempted suicide, or violent death. JAMA 2010;303:1401-9.

34 Schneeweiss S. Sensitivity analysis and external adjustment for unmeasured confounders in epidemiologic database studies of therapeutics. Pharmacoepidemiol Drug Saf 2006:15:291-303.

35 Liperoti R, Onder G, Landi F, Lapane K, Mor V, Bernabei R, et al. All-cause mortality associated with atypical and conventional antipsychotics among nursing home residents with dementia: a retrospective cohort study. J Clin Psych 2009;70:1340-7.

36 Rossom R, Rector T, Lederle F, Dysken M. Are all commonly prescribed antipsychotics associated with greater mortality in elderly male veterans with dementia? J Am Geriatr Soc 2010;58:1027-34

37 Setoguchi S, Wang P, Brookhart M, Canning C, Kaci L, Schneeweiss S. Potential causes of higher mortality in elderly users of conventional and atypical antipsychotic medications. J Am Geriatr Soc 2008;56:1644-50.

38 Maher A, Maglione M, Bagley S, Suttorp M, Hu J-H, Ewing B, et al. Efficacy and comparative effectiveness of atypical antipsychotic medications for off-label uses in adults: a systematic review and meta-analysis. JAMA 2011;306:1359-69.

39 Huybrechts K, Brookhart A, Rothman K, Silliman R, Gerhard T, Crystal S, et al. Comparison of different approaches to confounding adjustment in a study on the effect of antipsychotic medication on mortality in older nursing home patients. Am J Epidemiol 2011;174:1089-99.

40 Andreasen N, Pressler M, Nopoulos P, Miller D, Ho B. Antipsychotic dose equivalents and dose-years: a standardized method for comparing exposure to different drugs. Biol Psychiatry 2010;67:255-62.

41 Beck C. Psychosocial and behavioral interventions for Alzheimer's disease patients. Am $J$ Geriatr Psychiatry 1998;6:S41-8.

42 Snowden M, Sato K, Roy-Byrne P. Assessment and treatment of nursing home residents with depression or behavioral symptoms associated with dementia: a review of the literature. J Am Geriatr Soc 2003;51:1305-17.

43 Camp CJ, Cohen-Mansfield J, Capesuti EA. Use of nonpharmacologic interventions among nursing home residents with dementia. Psychiatr Serv 2002;53:1397-401.

44 Cummings J. Behavioral and neuropsychiatric outcomes in Alzheimer's disease. CNS Spectr 2005;10:22-5.

45 Sturmer T, Rothman KJ, Avorn J, Glynn RJ. Treatment effects in the presence of unmeasured confounding: dealing with observations in the tails of the propensity score distribution-a simulation study. Am J Epidemiol 2010;172:843-54.

Accepted: 29 December 2011

\section{Cite this as: BMJ 2012;344:e977}

This is an open-access article distributed under the terms of the Creative Commons Attribution Non-commercial License, which permits use, distribution, and reproduction in any medium, provided the original work is properly cited, the use is non commercial and is otherwise in compliance with the license. See: http://creativecommons.org/licenses/by$\mathrm{nc} / 2.0 /$ and http://creativecommons.org/licenses/by-nc/2.0/legalcode. 


\section{What is already known on this topic}

Both typical and atypical antipsychotics carry a black box warning of an increased risk of death in elderly patients with behavioural symptoms associated with dementia

Despite their known risks and the absence of compelling efficacy data, these drugs are still used widely in this population Little is known about whether different drugs differ in their mortality risk

\section{What this study adds}

In a large cohort of elderly patients in nursing homes, antipsychotic drugs conferred a dose related risk of death: compared with risperidone, haloperidol users had an increased risk and quetiapine users a decreased risk

The effects were strongest shortly after the start of treatment and remained after adjustment for dose

Though extensive measures were taken to mitigate confounding, and findings were consistent in sensitivity and confirmatory analyses, they should be confirmed with other data sources

\section{Tables}

Table 1 | Selected characteristics in residents starting antipsychotic drugs during stay in nursing home. Figures are numbers (percentage) or means

\begin{tabular}{|c|c|c|c|c|c|c|}
\hline & $\begin{array}{c}\text { Haloperidol } \\
(n=5904)\end{array}$ & $\begin{array}{c}\text { Aripiprazole } \\
(n=1849)\end{array}$ & $\begin{array}{c}\text { Olanzapine ( } \mathrm{n}=22 \\
919)\end{array}$ & $\begin{array}{c}\text { Quetiapine ( } \mathrm{n}=15 \\
776)\end{array}$ & $\begin{array}{c}\text { Risperidone }(\mathrm{n}=27 \\
936)\end{array}$ & $\begin{array}{l}\text { Ziprasidone } \\
(\mathrm{n}=1061)\end{array}$ \\
\hline High dose* & 2056/3507 (58.6) & $1501 / 1739(86.3)$ & $9461 / 21296(44.4)$ & 7376/14 694 (50.2) & 2498/25 $033(10.0)$ & 630/924 (68.2) \\
\hline \multicolumn{7}{|l|}{ Region: } \\
\hline Northeast & $648(11.0)$ & $332(18.0)$ & $3816(16.6)$ & 2801 (17.8) & $4577(16.4)$ & $47(4.4)$ \\
\hline Midwest & $1904(32.2)$ & $607(32.8)$ & $6582(28.7)$ & $4430(28.1)$ & $8749(31.3)$ & $253(23.8)$ \\
\hline South & $2531(42.9)$ & $764(41.3)$ & $8976(39.2)$ & $6952(44.1)$ & $11419(40.9)$ & $695(65.5)$ \\
\hline West & $821(13.9)$ & $146(7.9)$ & 3545 (15.5) & $1593(10.1)$ & 3191 (11.4) & $66(6.2)$ \\
\hline Rural ( $v$ urban) setting & $2508(42.5)$ & $519(28.1)$ & $7255(31.7)$ & $4753(30.1)$ & $9938(35.6)$ & $512(48.3)$ \\
\hline \multicolumn{7}{|l|}{ Demographics: } \\
\hline Male & $1659(28.1)$ & $508(27.5)$ & $5555(24.2)$ & $4028(25.5)$ & $6858(24.5)$ & $302(28.5)$ \\
\hline Mean age (years) & 83.8 & 81.9 & 83.5 & 83.0 & 83.7 & 82.6 \\
\hline White race & $4730(80.1)$ & $1473(79.7)$ & $18969(82.8)$ & $13015(82.5)$ & $22983(82.3)$ & $851(80.2)$ \\
\hline \multicolumn{7}{|l|}{ Psychiatric morbidity: } \\
\hline Dementia & $3281(55.6)$ & $1164(63.0)$ & $13418(58.5)$ & $9668(61.3)$ & $16899(60.5)$ & $655(61.7)$ \\
\hline Depression & $1407(23.8)$ & $701(37.9)$ & $6915(30.2)$ & $5251(33.3)$ & $7900(28.3)$ & $371(35.0)$ \\
\hline Anxiety & $168(2.8)$ & $42(2.3)$ & $752(3.3)$ & $524(3.3)$ & $811(2.9)$ & $45(4.2)$ \\
\hline Delirium & $373(6.3)$ & $132(7.1)$ & $1315(5.7)$ & $1169(7.4)$ & $1705(6.1)$ & $93(8.8)$ \\
\hline Psychotic disorder & $623(10.6)$ & $244(13.2)$ & $2400(10.5)$ & $1800(11.4)$ & $3053(10.9)$ & $165(15.6)$ \\
\hline \multicolumn{7}{|c|}{ Impairment in cognitive function: } \\
\hline Intact to moderate & $846(14.3)$ & $330(17.8)$ & $3578(15.6)$ & $2331(14.8)$ & $4049(14.5)$ & $180(17.0)$ \\
\hline Moderate to severe & $3252(55.1)$ & $1093(59.1)$ & $13036(56.9)$ & $8963(56.8)$ & $15960(57.1)$ & $616(58.1)$ \\
\hline Severe to very severe & $1806(30.6)$ & $426(23.0)$ & $6305(27.5)$ & $4482(28.4)$ & $7927(28.4)$ & $265(25.0)$ \\
\hline Delusions & $190(3.2)$ & $87(4.7)$ & $848(3.7)$ & $575(3.6)$ & $1050(3.8)$ & $41(3.9)$ \\
\hline $\begin{array}{l}\text { Verbally or physically } \\
\text { abusive behaviour }\end{array}$ & $810(13.7)$ & $270(14.6)$ & $3317(14.5)$ & $2154(13.7)$ & $4119(14.7)$ & $157(14.8)$ \\
\hline $\begin{array}{c}\text { Non-aggressive } \\
\text { behavioural problems }\end{array}$ & $1512(25.6)$ & $453(24.5)$ & $5908(25.8)$ & $4039(25.6)$ & $7385(26.4)$ & $28426.8)$ \\
\hline \multicolumn{7}{|l|}{ Cardiovascular morbidity: } \\
\hline Myocardial infarction & $417(7.1)$ & $85(4.6)$ & $1197(5.2)$ & $811(5.1)$ & $1587(5.7)$ & $48(4.5)$ \\
\hline Arrhythmias & $1617(27.4)$ & $373(20.2)$ & $5262(23.0)$ & $3674(23.3)$ & $6613(23.7)$ & $236(22.2)$ \\
\hline Ischaemic heart disease & $297(5.0)$ & $74(4.0)$ & $1046(4.6)$ & $626(4.0)$ & $1236(4.4)$ & $51(4.8)$ \\
\hline Hypertension & $3897(66.0)$ & $1262(68.3)$ & $14413(62.9)$ & $10439(66.2)$ & $18129(64.9)$ & $748(70.5)$ \\
\hline Congestive heart failure & $2286(38.7)$ & $562(30.4)$ & $7107(31.0)$ & $5015(31.8)$ & $9057(32.4)$ & $362(34.1)$ \\
\hline Cerebrovascular disease & $1752(29.7)$ & $532(28.8)$ & $6445(28.1)$ & $4546(28.8)$ & $7868(28.2)$ & $309(29.1)$ \\
\hline
\end{tabular}


Table 1 (continued)

\begin{tabular}{|c|c|c|c|c|c|c|}
\hline & $\begin{array}{l}\text { Haloperidol } \\
(n=5904)\end{array}$ & $\begin{array}{l}\text { Aripiprazole } \\
\quad(n=1849)\end{array}$ & $\begin{array}{c}\text { Olanzapine }(n=22 \\
919)\end{array}$ & $\begin{array}{c}\text { Quetiapine }(\mathrm{n}=15 \\
776)\end{array}$ & $\begin{array}{c}\text { Risperidone }(\mathrm{n}=27 \\
936)\end{array}$ & $\begin{array}{l}\text { Ziprasidone } \\
\qquad(n=1061)\end{array}$ \\
\hline \multicolumn{7}{|l|}{ Other comorbidities: } \\
\hline Diabetes & $1482(25.1)$ & $536(29.0)$ & $4699(20.5)$ & $3918(24.8)$ & $6656(23.8)$ & $266(25.1)$ \\
\hline Parkinson's disease & $270(4.6)$ & $112(6.1)$ & $1132(4.9)$ & $1525(9.7)$ & $1187(4.2)$ & $66(6.2)$ \\
\hline \multicolumn{7}{|l|}{ Functional impairment: } \\
\hline $\begin{array}{l}\text { Independent, supervision, } \\
\text { or limited }\end{array}$ & $2153(36.5)$ & $777(42.0)$ & $9222(40.2)$ & $6055(38.4)$ & $11211(40.1)$ & $445(41.9)$ \\
\hline Dependence or extensive & $3403(57.6)$ & $1012(54.7)$ & $12715(55.5)$ & $8982(56.9)$ & $15509(55.5)$ & $561(52.9)$ \\
\hline Total dependence & $348(5.9)$ & $60(3.2)$ & $982(4.3)$ & $739(4.7)$ & $1216(4.4)$ & $55(5.2)$ \\
\hline \multicolumn{7}{|c|}{ General indicators of comorbidity: } \\
\hline Mean Charlson index $†$ & 3.3 & 3.2 & 3.0 & 3.1 & 3.1 & 3.1 \\
\hline $\begin{array}{l}\text { Mean No of prescription } \\
\text { drugs received }\end{array}$ & 15.9 & 15.0 & 14.3 & 15.0 & 14.4 & 15.3 \\
\hline $\begin{array}{l}\text { Mean No of outpatient } \\
\text { visits }\end{array}$ & 1.1 & 0.8 & 0.9 & 0.9 & 0.9 & 1.1 \\
\hline $\begin{array}{l}\text { Mean No of days in } \\
\text { hospital }\end{array}$ & 20.0 & 15.3 & 17.9 & 17.7 & 18.0 & 20.6 \\
\hline \multicolumn{7}{|l|}{ History of prescriptions: } \\
\hline Antidepressants & 3617 (61.3) & $1351(73.1)$ & $15863(69.2)$ & $11362(72.0)$ & $18502(66.2)$ & $716(67.5)$ \\
\hline Hypnotic agents & $3042(51.5)$ & $802(43.4)$ & $10493(45.8)$ & $7623(48.3)$ & $12570(45.0)$ & $485(45.7)$ \\
\hline $\begin{array}{l}\text { Other psychoactive } \\
\text { agents } \neq\end{array}$ & $656(11.1)$ & $283(15.3)$ & $3078(13.4)$ & $2202(14.0)$ & 3335 (11.9) & $149(14.0)$ \\
\hline Dementia drug & $1496(25.3)$ & $766(41.4)$ & $6982(30.5)$ & $5742(36.4)$ & $9163(32.8)$ & $438(41.3)$ \\
\hline
\end{tabular}

* $>50$ mg chlorpromazine equivalents, in subset of patients receiving tablets or caplets.

†Individual comorbidities defined based on at least one admission to hospital or at least one outpatient visit with respective ICD codes.

łIncludes barbiturate, non-benzodiazepine anxiolytic, stimulant/ADHD drug, lithium, valproate, carbamazepine, lamotrigine. 


\begin{tabular}{|c|c|c|c|c|c|c|c|c|c|c|c|c|}
\hline & \multicolumn{2}{|c|}{$\begin{array}{c}\text { Haloperidol } \\
\text { ( } n=5904 ; 683 \text { person } \\
\text { years) }\end{array}$} & \multicolumn{2}{|c|}{$\begin{array}{c}\text { Aripiprazole } \\
(n=1849 ; 465 \\
\text { person years) }\end{array}$} & \multicolumn{2}{|c|}{$\begin{array}{c}\text { Olanzapine }(\mathrm{n}=22 \\
919 ; 5741 \text { person } \\
\text { years })\end{array}$} & \multicolumn{2}{|c|}{$\begin{array}{c}\text { Quetiapine ( } n=15 \\
776 ; 3945 \text { person } \\
\text { years) }\end{array}$} & \multicolumn{2}{|c|}{$\begin{array}{c}\text { Risperidone ( } n=27 \\
936 ; 6720 \text { person } \\
\text { years) }\end{array}$} & \multicolumn{2}{|c|}{$\begin{array}{c}\text { Ziprasidone } \\
(\mathrm{n}=1061 ; 235)\end{array}$} \\
\hline & No & $\begin{array}{l}\text { Rate }(95 \% \\
\quad \text { Cl) }\end{array}$ & No & $\begin{array}{l}\text { Rate }(95 \% \\
\quad \mathrm{Cl})\end{array}$ & No & $\begin{array}{c}\text { Rate }(95 \% \\
\mathrm{Cl})\end{array}$ & No & $\begin{array}{c}\text { Rate }(95 \% \\
\text { Cl) }\end{array}$ & No & $\begin{array}{l}\text { Rate }(95 \% \\
\quad \text { Cl) }\end{array}$ & No & $\begin{array}{l}\text { Rate }(95 \% \\
\quad \mathrm{Cl})\end{array}$ \\
\hline $\begin{array}{l}\text { All non-cancer } \\
\text { mortality }\end{array}$ & 745 & $\begin{array}{c}109.1(101.4 \\
\text { to } 117.0)\end{array}$ & 122 & $\begin{array}{c}26.2(21.8 \\
\text { to } 31.1)\end{array}$ & 2104 & $\begin{array}{c}36.7(35.1 \\
\text { to } 38.2)\end{array}$ & 1120 & $\begin{array}{c}28.4(26.8 \\
\text { to } 30.1)\end{array}$ & 2434 & $\begin{array}{c}36.2(34.8 \\
\text { to } 37.7)\end{array}$ & 73 & $\begin{array}{c}31.1(24.4 \\
\text { to } 38.6)\end{array}$ \\
\hline \multicolumn{13}{|c|}{ Cause specific mortality: } \\
\hline $\begin{array}{l}\text { Circulatory } \\
\text { system }\end{array}$ & 351 & $\begin{array}{c}51.4(46.2 \text { to } \\
56.9)\end{array}$ & 57 & $\begin{array}{c}12.3(9.3 \text { to } \\
15.6)\end{array}$ & 1045 & $\begin{array}{c}18.2(17.1 \\
\text { to } 19.3)\end{array}$ & 542 & $\begin{array}{c}13.7(12.6 \\
\text { to } 14.9)\end{array}$ & 1230 & $\begin{array}{c}18.3(17.3 \\
\text { to } 19.3)\end{array}$ & 37 & $\begin{array}{c}15.8(11.1 \\
\text { to } 21.2)\end{array}$ \\
\hline $\begin{array}{l}\text { Cerebrovascular } \\
\text { diseases }\end{array}$ & 81 & $\begin{array}{c}11.9(9.4 \text { to } \\
14.6)\end{array}$ & 6 & $\begin{array}{c}1.3(0.5 \text { to } \\
2.5)\end{array}$ & 192 & $\begin{array}{c}3.3(2.9 \text { to } \\
3.8)\end{array}$ & 102 & $\begin{array}{c}2.6(2.1 \text { to } \\
3.1)\end{array}$ & 263 & $\begin{array}{c}3.9(3.5 \text { to } \\
4.4)\end{array}$ & 11 & $\begin{array}{c}4.7 \text { (2.3 to } \\
7.9)\end{array}$ \\
\hline $\begin{array}{l}\text { Respiratory } \\
\text { system }\end{array}$ & 129 & $\begin{array}{c}18.9(15.8 \text { to } \\
22.3)\end{array}$ & 26 & $\begin{array}{c}5.6(3.6 \text { to } \\
7.9)\end{array}$ & 320 & $\begin{array}{c}5.6(5.0 \text { to } \\
6.2)\end{array}$ & 151 & $\begin{array}{c}3.8 \text { (3.2 to } \\
4.5)\end{array}$ & 342 & $\begin{array}{l}5.1(4.6 \text { to } \\
5.6)\end{array}$ & 13 & $\begin{array}{c}5.5(2.9 \text { to } \\
9.0)\end{array}$ \\
\hline Other & 265 & $\begin{array}{c}38.8(34.3 \text { to } \\
43.6)\end{array}$ & 39 & $\begin{array}{c}8.4(6.0 \text { to } \\
11.2)\end{array}$ & 739 & $\begin{array}{l}12.9(12.0 \\
\text { to } 13.8)\end{array}$ & 427 & $\begin{array}{c}10.8(9.8 \text { to } \\
11.9)\end{array}$ & 862 & $\begin{array}{l}12.8(12.0 \\
\text { to } 13.7)\end{array}$ & 23 & $\begin{array}{c}9.8(6.2 \text { to } \\
14.2)\end{array}$ \\
\hline
\end{tabular}

*Rate expressed per 100 person years. 


\begin{tabular}{|c|c|c|c|c|c|}
\hline & \multirow[b]{2}{*}{ No of events } & \multicolumn{4}{|c|}{ HR $(95 \% \mathrm{Cl})$} \\
\hline & & Unadjusted & $\begin{array}{l}\text { Adjusted for age, sex, and } \\
\text { calendar year }\end{array}$ & $\begin{array}{l}\text { Adjusted for propensity } \\
\text { score* }\end{array}$ & $\begin{array}{c}\text { Adjusted for high } \\
\text { dimensional propensity } \\
\text { score }^{*}\end{array}$ \\
\hline \multicolumn{6}{|c|}{ Non-cancer mortality } \\
\hline Haloperidol & 745 & 2.42 (2.21 to 2.65$)$ & 2.37 (2.17 to 2.59$)$ & 2.07 (1.89 to 2.26$)$ & 1.81 (1.65 to 1.98$)$ \\
\hline Aripiprazole & 122 & 0.76 (0.63 to 0.92$)$ & 0.77 (0.64 to 0.93$)$ & $0.88(0.73$ to 1.07$)$ & 0.95 (0.78 to 1.15$)$ \\
\hline Olanzapine & 2104 & 1.01 (0.95 to 1.07$)$ & $1.03(0.97$ to 1.09$)$ & $1.02(0.96$ to 1.08$)$ & 1.01 (0.95 to 1.08$)$ \\
\hline Quetiapine & 1120 & $0.80(0.74$ to 0.86$)$ & $0.80(0.75$ to 0.87$)$ & 0.81 (0.75 to 0.88$)$ & 0.83 (0.77 to 0.89$)$ \\
\hline Ziprasidone & 73 & 0.88 (0.69 to 1.12$)$ & $0.90(0.70$ to 1.14$)$ & $0.92(0.72$ to 1.17$)$ & 0.90 (0.69 to 1.17$)$ \\
\hline \multicolumn{6}{|c|}{ Cause specific mortality } \\
\hline \multicolumn{6}{|c|}{ Circulatory system: } \\
\hline Haloperidol & 351 & 2.25 (1.98 to 2.56$)$ & 2.21 (1.94 to 2.51 ) & 1.86 (1.63 to 2.12$)$ & 1.66 (1.46 to 1.90$)$ \\
\hline Aripiprazole & 57 & 0.71 (0.54 to 0.93$)$ & 0.75 (0.57 to 0.98$)$ & $0.84(0.64$ to 1.11$)$ & $0.92(0.69$ to 1.22$)$ \\
\hline Olanzapine & 1045 & $1.00(0.92$ to 1.09$)$ & 1.00 (0.92 to 1.09$)$ & $1.01(0.92$ to 1.10$)$ & $1.00(0.92$ to 1.09$)$ \\
\hline Quetiapine & 542 & 0.77 (0.70 to 0.86$)$ & 0.79 (0.71 to 0.88$)$ & $0.81(0.72$ to 0.89$)$ & 0.84 (0.75 to 0.93$)$ \\
\hline Ziprasidone & 37 & 0.85 (0.6 to 1.21$)$ & 0.89 (0.63 to 1.26$)$ & 0.89 (0.63 to 1.26$)$ & 0.91 (0.64 to 1.31$)$ \\
\hline \multicolumn{6}{|c|}{ Cerebrovascular diseases: } \\
\hline Haloperidol & 81 & 2.59 (2.00 to 3.37$)$ & 2.56 (1.97 to 3.33$)$ & 2.23 (1.71 to 2.90$)$ & 1.95 (1.48 to 2.58 ) \\
\hline Aripiprazole & 6 & $0.30(0.12$ to 0.72$)$ & 0.31 (0.13 to 0.76$)$ & $0.34(0.14$ to 0.82$)$ & $0.43(0.18$ to 1.06$)$ \\
\hline Olanzapine & 192 & $0.88(0.72$ to 1.06$)$ & $0.87(0.72$ to 1.06$)$ & 0.88 (0.73 to 1.07$)$ & $0.83(0.68$ to 1.01$)$ \\
\hline Quetiapine & 102 & $0.66(0.52$ to 0.84$)$ & 0.68 (0.54 to 0.87$)$ & 0.67 (0.53 to 0.86$)$ & 0.73 (0.57 to 0.93$)$ \\
\hline Ziprasidone & 11 & $0.98(0.48$ to 1.98$)$ & 1.05 (0.52 to 2.12 ) & $1.03(0.50$ to 2.10$)$ & 1.01 (0.47 to 2.17$)$ \\
\hline \multicolumn{6}{|c|}{ Respiratory system: } \\
\hline Haloperidol & 129 & 3.06 (2.45 to 3.82$)$ & 3.01 (2.41 to 3.76$)$ & 2.53 (2.02 to 3.18$)$ & 1.99 (1.57 to 2.51 ) \\
\hline Aripiprazole & 26 & $1.11(0.73$ to 1.68$)$ & $1.07(0.70$ to 1.63$)$ & 1.29 (0.85 to 1.97$)$ & $1.36(0.90$ to 2.07$)$ \\
\hline Olanzapine & 320 & 1.07 (0.91 to 1.25$)$ & 1.08 (0.93 to 1.27$)$ & 1.05 (0.89 to 1.23$)$ & $1.00(0.85$ to 1.17$)$ \\
\hline Quetiapine & 151 & 0.76 (0.63 to 0.93$)$ & 0.74 (0.61 to 0.90$)$ & 0.76 (0.62 to 0.93$)$ & 0.70 (0.57 to 0.86$)$ \\
\hline Ziprasidone & 13 & $1.14(0.64$ to 2.03$)$ & $1.12(0.63$ to 2.00$)$ & 1.19 (0.66 to 2.13$)$ & $1.03(0.56$ to 1.89$)$ \\
\hline \multicolumn{6}{|l|}{ Other: } \\
\hline Haloperidol & 265 & 2.42 (2.09 to 2.80$)$ & 2.36 (2.04 to 2.74$)$ & 2.19 (1.88 to 2.55$)$ & 1.95 (1.67 to 2.28 ) \\
\hline Aripiprazole & 39 & $0.70(0.50$ to 0.96$)$ & $0.69(0.50$ to 0.96$)$ & 0.78 (0.56 to 1.09$)$ & $0.77(0.54$ to 1.10$)$ \\
\hline Olanzapine & 739 & $1.01(0.91$ to 1.12$)$ & $1.04(0.94$ to 1.15$)$ & $1.02(0.92$ to 1.13$)$ & $1.03(0.92$ to 1.14$)$ \\
\hline Quetiapine & 427 & 0.85 (0.75 to 0.96$)$ & 0.84 (0.75 to 0.95$)$ & 0.85 (0.75 to 0.95$)$ & $0.84(0.74$ to 0.95$)$ \\
\hline Ziprasidone & 23 & $0.82(0.54$ to 1.25$)$ & $0.81(0.53$ to 1.24$)$ & $0.86(0.56$ to 1.31$)$ & 0.78 (0.48 to 1.26$)$ \\
\hline
\end{tabular}

*Models stratified across 10 th of propensity score, after truncating $2.5 \%$ of patients on either extreme of propensity score distribution in each exposure pairing to avoid bias from inclusion of people with propensity score from outside shared range of scores. ${ }^{45}$ 


\section{Figures}

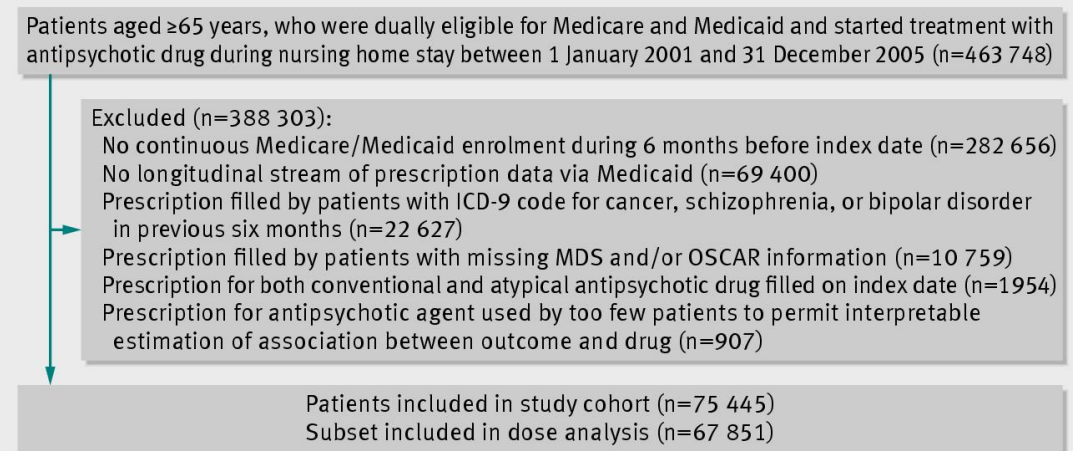

Fig 1 Assembly of study cohort of patients in nursing homes starting treatment with antipsychotic drugs (ICD-9=international classification of diseases, ninth revision; MDS=Minimum Data Set; OSCAR=Online Survey Certification and Reporting) 

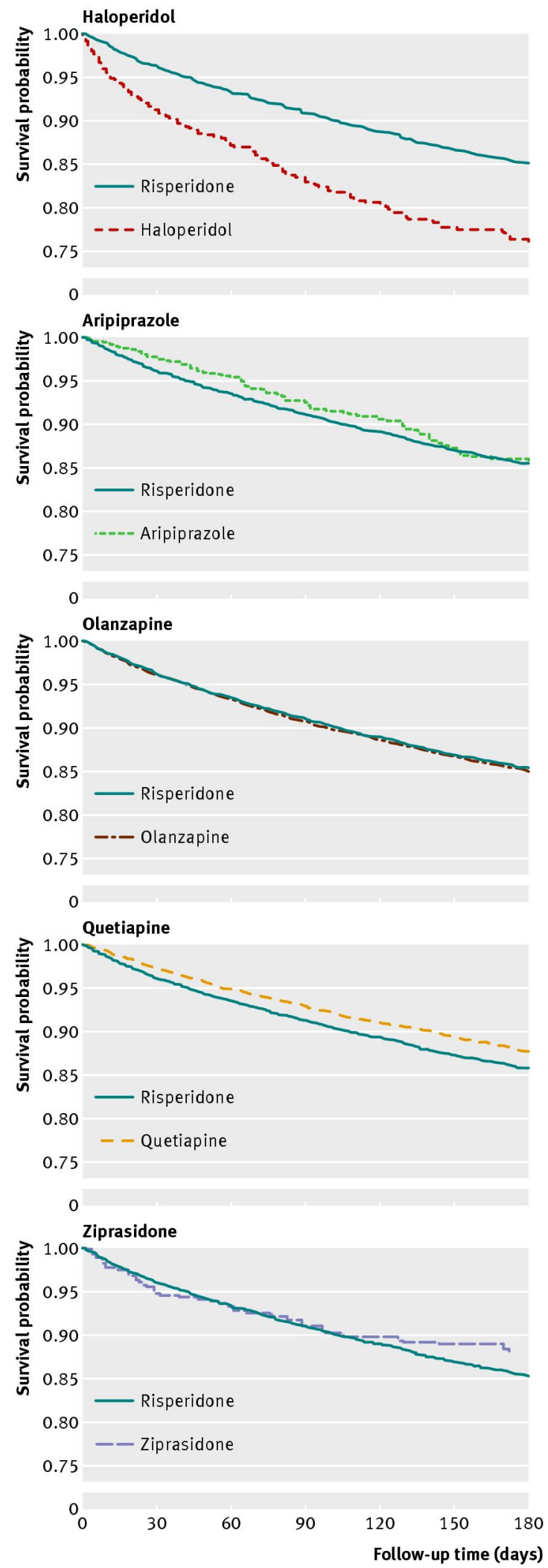

Fig 2 Adjusted Kaplan-Meier plots for death from causes other than cancer. Each participant is weighed by inverse of his or her probability for treatment as estimated in multivariate propensity score analysis 


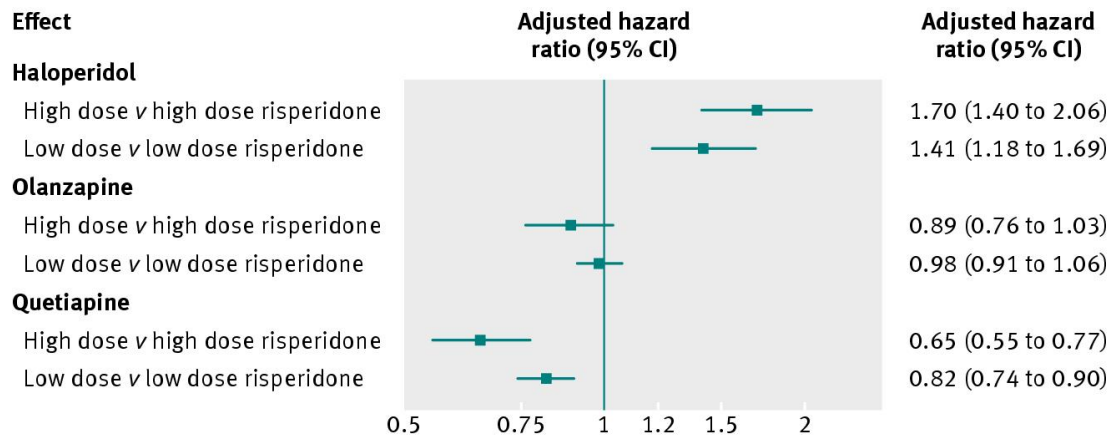

Fig 3 Hazard ratios (adjusted for propensity score) for death from causes other than cancer by dose of various antipsychotic drugs compared with similar dose of risperidone (restricted to users of tablets or caplets). Results for aripiprazole and ziprasidone not presented because of small numbers of events in some dose groups

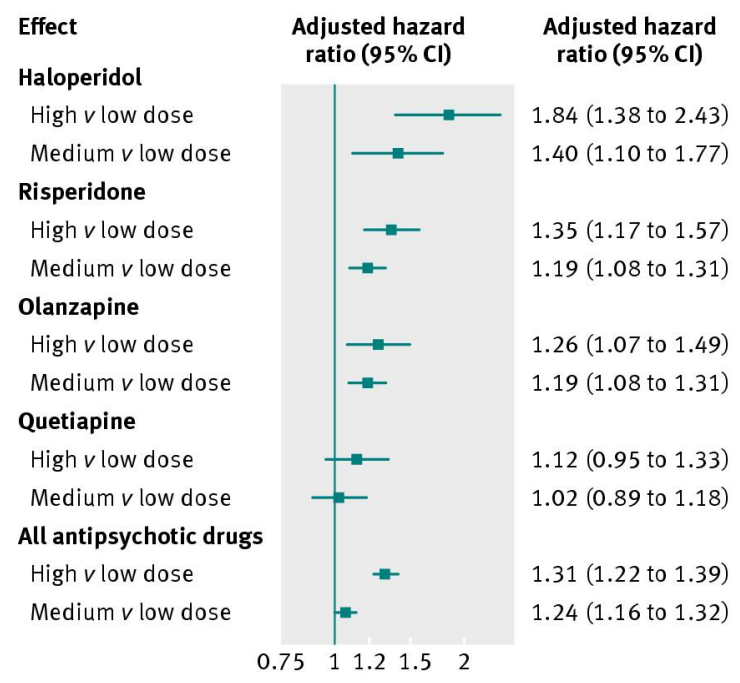

Fig 4 Hazard ratios (adjusted propensity score) for death from causes other than cancer by dose of various antipsychotic drugs with low dose group of each drug as reference. Results for aripiprazole and ziprasidone not presented because of small numbers of events in some dose groups 